\title{
GLASSY STATES IN A SHAKEN SANDBOX
}

\author{
PETER F. STADLER \\ Institut für Theoretische Chemie und Molekulare Strukturbiologie, \\ Universität Wien, Währingerstraße 17, A-1090 Wien, Austria; and \\ The Santa Fe Institute, 1399 Hyde Park Rd., Santa Fe NM 87501, USA \\ ANITA MEHTA \\ S N Bose National Centre for Basic Sciences, Block JD Sector 3, Salt Lake, Calcutta 700098, \\ India; and \\ ICTP, Strada Costiera 11, I-34100 Trieste, Italy \\ JEAN-MARC LUCK \\ Service de Physique Théorique (URA 2306 of CNRS), CEA Saclay, \\ 91191 Gif-sur-Yvette cedex, France \\ Received () \\ Revised ()
}

\begin{abstract}
Our model of shaken sand, presented in earlier work, has been extended to include a more realistic 'glassy' state, i.e., when the sandbox is shaken at very low intensities of vibration. We revisit some of our earlier results, and compare them with our new results on the revised model. Our analysis of the glassy dynamics in our model shows that a variety of ground states is obtained; these fall in two categories, which we argue are representative of regular and irregular packings.
\end{abstract}

\section{Introduction}

The test of a good lattice model of a complex system is whether it succeeds in capturing the essential physics of a real system in its bid to reduce its technical complexity. Areas as diverse as traffic flow [10, plate tectonics [12,13] and granular flow [28] are examples where lattice models have been used rather successfully despite their apparent simplicity, to describe at least a few of the salient features of some genuinely complex systems.

In this spirit, we present two versions of a model of shaken sand in the following; both models exhibit behaviour that is representative of shaken sand between the fluidised and the glassy regimes. While the second model includes rather complex interactions between the 'grains' in the frozen state, in contrast to the first, the latter is nevertheless surprisingly successful in replicating at least some of the qualitative features associated with the glassy regime. One of the aims of this contribution is then to identify some of the essential features that are needed for a (discrete) 
minimal model of the system in question.

The earlier model ('old model') [32] is the generalisation of a cellular-automaton (CA) model [4] 28] of an avalanching sandpile. This model shows both fast and slow dynamics in the appropriate regimes: in particular, it reduces to an exactly solvable model of noninteracting grains in the frozen ('glassy') regime, and provides one with a toy model for ageing in vibrated sand 9,24$]$.

Next, we present a more realistic version of the model ('new model') [26], which is the topic of ongoing research. While identical in the fluidised regime, the latter model is less of a toy model for the glassy state, in that the grains are no longer noninteracting, but are coupled to each other based on their orientations. Our analysis shows that a rich variety of ground states is obtained, which we analyse in terms of a particular parameter that has an interpretation in terms of the irregularity of the grains.

\section{The Model}

We consider a rectangular lattice of height $H$ and width $W$ with $N \leq H W$ grains located at the lattice points, shaken with vibration intensity $\Gamma$. Each grain is a rectangle with sides 1 and $a \leq 1$, respectively. Consider a grain $(i, j)$ in row $i$ and column $j$ whose height at any given time is given by $h_{i j}=n_{i j-}+a n_{i j+}$, where $n_{i j-}$ is the number of vertical grains and $n_{i j+}$ is the number of horizontal grains below $(i, j)$.

The dynamics of the model are described by the following rules:

(i) If lattice sites $(i+1, j-1),(i+1, j)$, or $(i+1, j+1)$ are empty, grain $(i, j)$ moves there with a probability $\exp (-1 / \Gamma)$, in units such that the acceleration due to gravity, the mass of a grain, and the height of a lattice cell all equal unity.

(ii) If the lattice site $(i-1, j)$ below the grain is empty, it will fall down.

(iii) If lattice sites $(i-1, j \pm 1)$ are empty, the grain at height $h_{i j}$ will fall to either lower neighbour, provided the height difference $h_{i j}-h_{i-1, j \pm 1} \geq 2$.

(iv) The grain flips from horizontal to vertical with probability $\exp \left(-m_{i j}(\Delta H+\right.$ $\Delta h) / \Gamma$ ), where $m_{i j}$ is the mass of the pile (consisting of grains of unit mass) above grain $(i, j)$. For a rectangular grain, $\Delta H=1-a$ is the height difference between the initial horizontal and the final vertical state of the grain. Similarly, the activation energy for a flip reads $\Delta h=b-1$, where $b=\sqrt{1+a^{2}}$ is the diagonal length of a grain.

(v) The grain flips from vertical to horizontal with probability $\exp \left(-m_{i j} \Delta h / \Gamma\right)$.

These rules yield a rich variety of dynamical behaviour, depicted in Fig. 11, which shows the time evolution of the sandbox under the effect of different vibrational intensities. All the boxes are started in the same initial state; the observed behaviours correspond to the glassy regime (top row) and the fluidised regime (bottom row). In the intermediate regime separating the two [32], individual-particle relaxations 

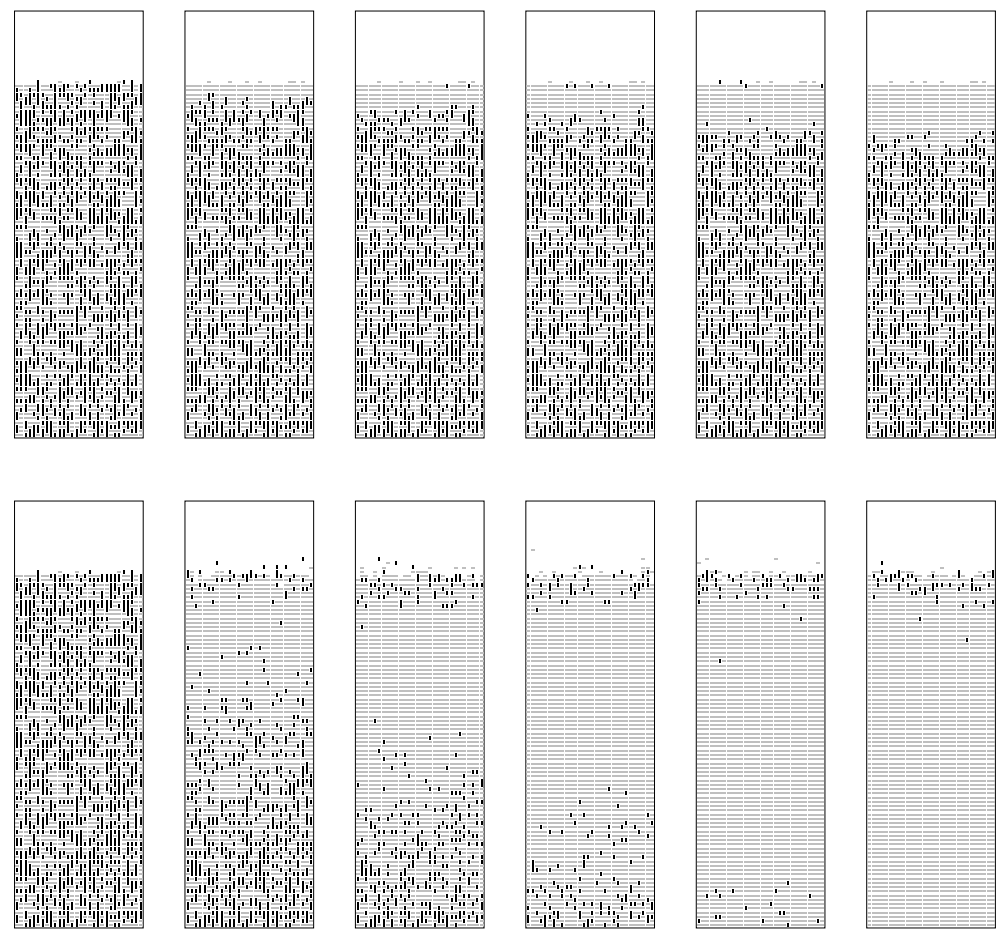

Fig. 1. Snapshots of a sandbox with $W=30, H=100, n=2500$ grains with $a=0.7, \Delta h=0.05$, and shaking intensities $\Gamma=0.1$ (top row: glassy regime) and 0.8 (bottom row: fluidised regime), for times $t=1$ (before tapping), $t=2$ (each grain on average touched once by the MC simulation), $t=5,10,30$, and 100 .

are the mechanism for each particle to find local stability. In related work [7,8], this was associated with a threshold, called the single particle relaxation threshold (SPRT).

In line with recent investigations of compaction [1,2, 2, 11, 16, 19, 20, 27, 30,31, we have examined the behaviour of the packing fraction of our model, as a function of the vibration intensity $\Gamma$. Let $N^{-}$and $N^{+}$be the numbers of vertical and horizontal grains in the box. The packing fraction $\phi$ is:

$$
\phi=\frac{N^{+}-a N^{-}}{N^{+}+a N^{-}},
$$

which we use as an order parameter reflective of the behaviour of the compactivity [19,20]. The vertical orientation of a grain thus wastes space proportional to $1-a$, relative to the horizontal one.

The advantage of our current model is that at least conceptually it can be extended to non-rectangular grain shapes. In this sense, we can and will consider $a$, $\Delta H$, and $\Delta h$ as phenomenological parameters in the following. 


\section{A Spin-Model for the Ordered Regime}

The frozen regime is characterised by an absence of holes within the sandbox, and negligible surface roughness. Here, the earlier model [32] reduces to an exactly solvable model of $W$ independent columns of $H$ noninteracting grain orientations $\sigma_{n}(t)= \pm 1$, with $\sigma=+1$ denoting a horizontal grain, and $\sigma=-1$ denoting a vertical grain. The orientation of the grain at depth $n$, measured from the top of the system, evolves according to a continuous-time Markov dynamics, with rates

$$
\left\{\begin{array}{l}
w(-\rightarrow+)=\exp (-n \Delta h / \Gamma) \\
w(+\rightarrow-)=\exp (-n(\Delta H+\Delta h) / \Gamma),
\end{array}\right.
$$

as $m_{i j}=n=H+1-i$. The parameters $\Delta H$ and $\Delta h$ correspond to two characteristic lengths of the model,

$$
\xi_{\mathrm{eq}}=\Gamma / \Delta H, \quad \xi_{\mathrm{dyn}}=\Gamma / \Delta h .
$$

The equilibrium length $\xi_{\text {eq }}$ is the typical depth below which all grains are frozen into their horizontal ground-state orientation, while the dynamical length $\xi_{\text {dyn }}$ is characteristic of the divergence of the relaxation time with $\operatorname{depth}, \tau \sim \exp \left(n / \xi_{\text {dyn }}\right)$. As a consequence, any perturbation propagates only logarithmically slowly down the system, over an ordering length $\Lambda(t) \approx \xi_{\text {dyn }} \ln t$.

The detailed analysis of this model in earlier work, despite its 'toy model' nature, was remarkably successful in reproducing certain features of the glassy state. In this work, we present an improved version where the spins are no longer noninteracting, in the frozen regime. They are in fact coupled in a rather complex way; these more realistic interactions lead to a rich variety of ground states depending on the analogue of the aspect ratio $a$, which we argue is representative of the shape of the grains. We present this model in the next section.

\section{A Generalisation}

The generalisation of our earlier model involves the insertion of eq. (2.1) into the transition rates of the system. We thus require that, for a given value of $a$, the transitions are such that the packing fraction of the system is locally minimised. We now allow $a$ to take arbitrary values (while always remaining positive): $1-a$ can then be visualised as the size of the 'void' associated with the 'wrong' orientation of the grain. For convenience, we write these rules in a more general form below.

Thus, the dynamical rules (iv) and (v) of the rectangular grain model may be regarded as a special case of a more general model with transition rates

$$
\left\{\begin{array}{l}
w(-\rightarrow+)=\exp \left(-\left(\lambda_{i j}+\eta_{i j}\right) / \Gamma\right), \\
w(+\rightarrow-)=\exp \left(-\left(\lambda_{i j}-\eta_{i j}\right) / \Gamma\right),
\end{array}\right.
$$

where

$$
\eta_{i j}=A m_{i j+}+B m_{i j-}, \quad \lambda_{i j}=C m_{i j+}+D m_{i j-},
$$


are, respectively, the ordering field and the activation energy felt by grain $(i j)$. In these expressions, $m_{i j \pm}$ is the number of horizontal and vertical grains above the grain $(i, j)$, respectively. Note that grains are only counted from $(i, j)$ to the first void above level $j$. Thus $m_{i j}=m_{i j+}+m_{i j-}$.

The earlier model is recovered by setting

$$
A=B=\Delta H / 2, \quad C=D=\Delta H / 2+\Delta h .
$$

In the general situation where the equalities $A=B, C=D$ are not obeyed, the rates (4.1) depend on the orientations of all the grains above the grain under consideration. Our new model is therefore a fully directed model of interacting grains, where causality acts both in time and in space, as the orientation of a given grain only influences the grains below it and at later times.

The key parameter which governs the statics and dynamics of the model at low shaking intensity turns out to be the dimensionless ratio $\varepsilon=A / B$. Consider the ordered regime, where there are no holes, in the zero-temperature limit $(\Gamma \rightarrow 0)$. In this regime, the steady-state values of the grain orientations are given by the deterministic, recursive equation

$$
\sigma_{n}=\operatorname{sign}\left(\varepsilon n_{+}(n)+n_{-}(n)\right),
$$

where $n_{ \pm}(n)$ is the number of horizontal and vertical grains at depths $0, \ldots, n-1$, so that $n_{+}(n)+n_{-}(n)=n=1,2, \ldots$ We assume $\sigma_{0}=+1$.

As long as $\varepsilon \geq 0$, (4.4) leads to the trivial ground state where all the grains are horizontal $\left(\sigma_{n}=+1\right)$, generalising thus the case of the earlier model $(\varepsilon=1)$.

In the frustrated regime $(\varepsilon<0)$, ground states have a richer structure. They contain non-trivial fractions of horizontal and vertical grains,

$$
f_{+}=1 /(1-\varepsilon), \quad f_{-}=-\varepsilon /(1-\varepsilon) .
$$

The grain orientations are distributed in a way which depends, rather unexpectedly, on whether $\varepsilon$ is rational or not.

- Rational case: if $\varepsilon=-p / q$ is a negative rational number (in irreducible form), (4.4) determines the grain orientation $\sigma_{n}$ whenever the depth $n$ is not a multiple of $r=p+q$. The orientations of the latter grains are left free. We thus obtain an extensively degenerate set of ground states, each of them being a random sequence of two types of $r$-mers, i.e., clusters of $r$ grains. The associated configurational entropy per grain reads $\Sigma=(\ln 2) / r$. The simplest example is $\varepsilon=-1$, hence $r=2$ and $f_{-}=1 / 2$, where the clusters are +- and -+ , so that the ground states are all the dimerised grain configurations. Two examples consist of trimers $(r=3)$, namely $\varepsilon=-1 / 2$, with $f_{-}=1 / 3$ and clusters +-+ and -++ , and $\varepsilon=-2$, with $f_{-}=2 / 3$ and clusters +-- and -+- .

- Irrational case: if $\varepsilon$ is a negative irrational number, (4.4) determines all the grain orientations, so that the model admits a unique, non-degenerate 
ground state, where the grain orientations are distributed in a quasiperiodic fashion. The rule (4.4) is indeed equivalent to the cut-and-project algorithm used to build quasiperiodic binary chains, which are one-dimensional analogues of perfect quasicrystals $15,17,18,21,22,23,25]$. For instance, for $-\varepsilon=(\sqrt{5}-1) / 2 \approx 0.618033$ (the inverse golden mean), the unique ground state of the model is given by the well-known Fibonacci sequence:

$$
+-+-++-+-++-++-+-++-+-++-++-+-+\cdots
$$

\section{Numerical Results}

In Fig. 2 we show the behaviour of the packing fraction as a function of $\Gamma$, the shaking intensity, for both models described above for a square box of side 120, containing 10000 grains of unit mass, each of which has an aspect ratio of 0.7.

We note that the overall behaviour of the two models is rather similar, although the complexity of the second makes it far more prone to fluctuations even after the steady state has been reached. In each case, we observe

- a fluidised region: for $\Gamma \gg 1$, we observe an initial increase (caused by a non-equilibrium and transient 'ordering' of grains in the boundary layer) of the packing fraction that quickly relaxes to the equilibrium values $\phi_{\infty}$ in each case. This over-shooting effect in Fig. 2 increases with $\Gamma$, since grains ever deeper in the sandbox can now overcome their activation energy to relax to the horizontal.

- an intermediate region (for $\Gamma \approx 1$ ), where the packing fraction remains approximately constant in the bulk, while the surface equilibrates via the fast dynamics of single-particle relaxation. The specific $\phi_{\infty}$ at which this occurs, is the single-particle relaxation threshold density observed in Ref. [8]; nonequilibrium, non-ergodic, fast dynamics allows single particles locally to find their equilibrium configurations at this density. Analogous effects have been observed in recent experiments on colloids [35], where the correlated dynamics of fast particles was seen to be responsible for most relaxational behaviour before the onset of the glass transition.

- a frozen region (for $\Gamma \ll 1$ ), where the slow dynamics of the system results in a logarithmic growth of packing fraction with time:

$$
\phi-\phi_{\infty}=b(\Gamma) \ln t+a,
$$

where $b(\Gamma)$ increases with $\Gamma$, in good agreement with experiment 30,31. The slow dynamics has been identified [8] with a cascade process, where the free volume released by the relaxation of one or more grains allows for the ongoing relaxation of other grains in an extended neighbourhood. It includes the phenomenon of bridge collapse, which, for low vibration intensities, has been seen to be a major mechanism of compaction [2, 3, 27]. As $\Gamma$ decreases, the corresponding $\phi_{\infty}$ increases asymptotically towards the 

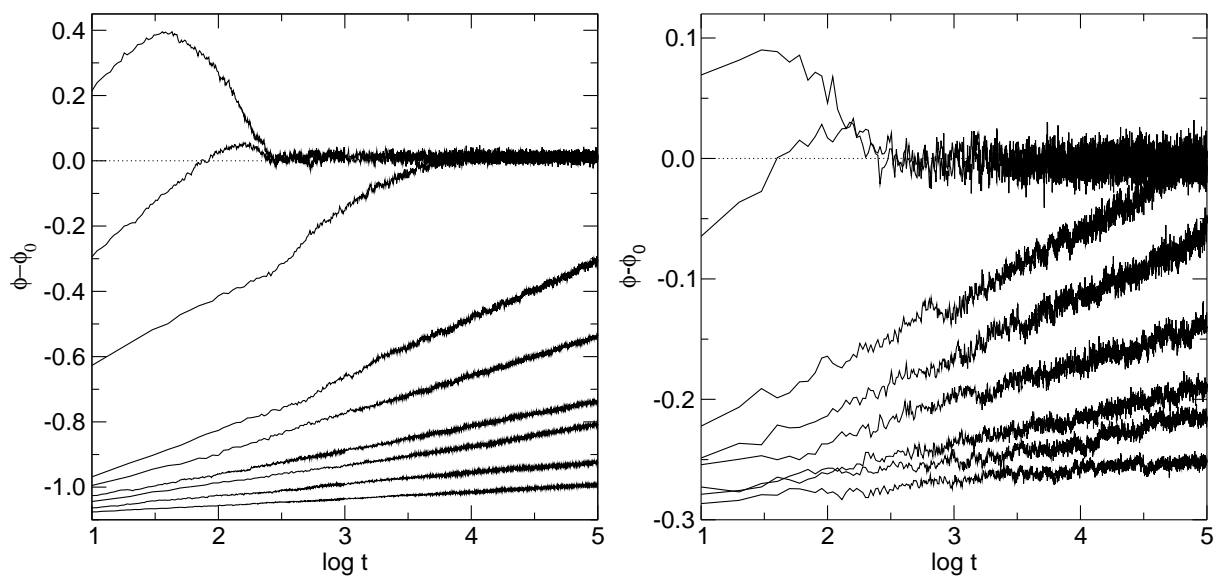

Fig. 2. Behaviour of packing fraction for both models described in text, with an aspect ratio of 0.7 in each case. For the old model (left), $A=B=0.35, C=D=0.4$, while for the new model (right) $A=-0.2, B=C=D=0.4$. The shaking intensities are, for bottom to top, $\Gamma=0.3$ (old model only), $0.5,0.8,1,1.5,2,5$, and 10 .

jamming limit $\phi_{\mathrm{jam}}$, identified with a dynamical phase transition in related work [6].

While we have presented in earlier work [32] a full analysis of two-time correlation functions for the old model, work is currently in progress to investigate this in the rather more complex new model.

We finally investigate the analogue of 'annealed cooling', where $\Gamma$ is increased and decreased cyclically, and the response of the packing fraction observed 30, 31. The results obtained here are similar to those [5] seen using more realistic models of shaken spheres, but the simplicity of the present lattice-based models allows for a greater transparency.

Starting with the sand in a fluidised state, as in the experiment [30,31, we submit the sandbox to taps at a given intensity $\Gamma$ for a time $t_{\text {tap }}$ and increase the intensity in steps of $\delta \Gamma$; at a certain point, the cycle is reversed, to go from higher to lower intensities. The entire process is then iterated twice. Fig. 3 shows the resulting behaviour of the volume fraction $\phi$ as a function of $\Gamma$, where an 'irreversible' branch and a 'reversible' branch of the compaction curve are seen, which meet at the 'irreversibility point' $\Gamma^{*}$ 30,31. The left- and right-hand side of Fig. 3 correspond respectively to high and low values of the 'ramp rate' $\delta \Gamma / t_{\text {tap }}$ 30, 31], while the upper and lower panels correspond respectively to the old and new versions of our model. As the ramp rate is lowered, we note that:

- the width of the hysteresis loop in the so-called reversible branch decreases, in both cases. The 'reversible' branch is thus not reversible at all; more realistic simulations of shaken spheres [2,3,27] confirm the first-order, irreversible nature of the transition, which allows the density to attain values 

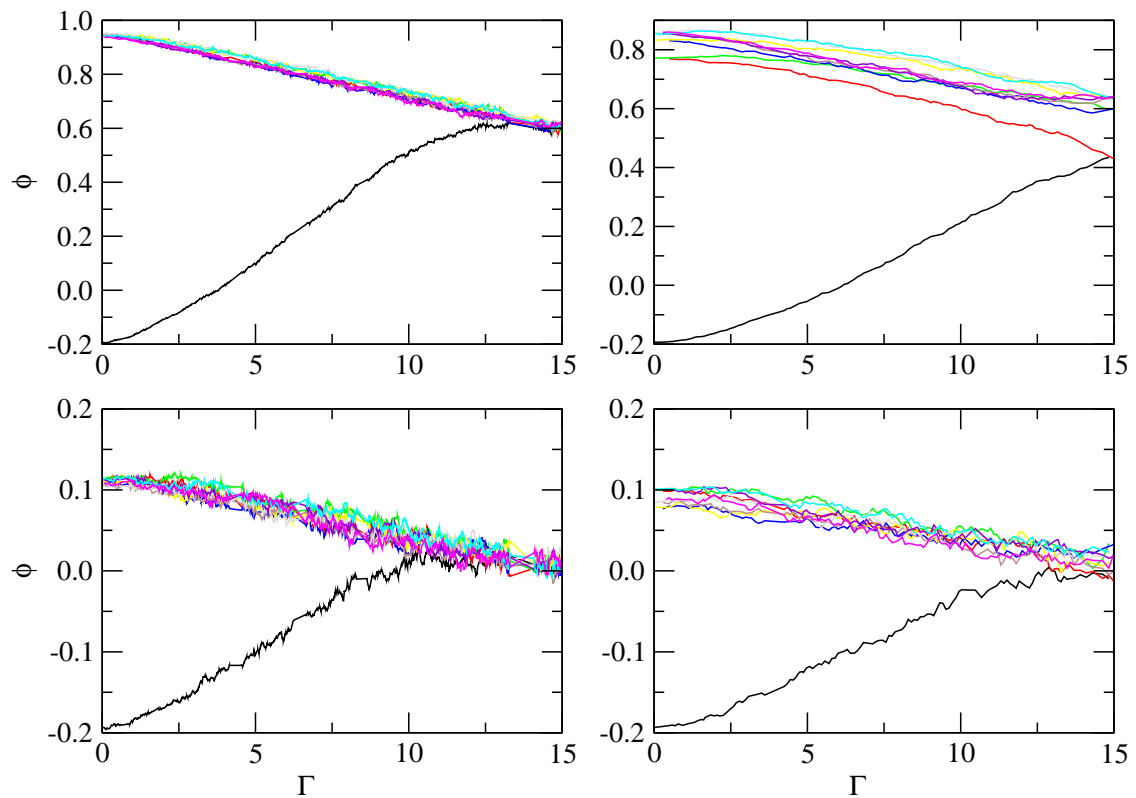

Fig. 3. Hysteresis curves. Top row: old model. Bottom row: new model. Left: $\delta \Gamma=0.1, t_{\text {tap }}=$ 2000 time units. Right: $\delta \Gamma=0.001, t_{\mathrm{tap}}=10^{5}$ time units. Note the approach of the irreversibility point $\Gamma^{*}$ to the 'shoulder' $\Gamma_{\text {jam }}$, as the ramp rate $\delta \Gamma / t_{\text {tap }}$ is lowered. The packing fraction tends to its close-packing limit in the limit of low intensities for the old model, while it asymptotes towards the jamming limit for the new model (see text).

that are substantially higher than random close packing, and quite close to the crystalline limit [29]. Precisely such a transition has also recently been observed experimentally in the compaction of rods [34].

- In both panels, the 'irreversibility point' $\Gamma^{*}$ approaches $\Gamma_{\text {jam }}$ (the shaking intensity at which the jamming limit $\phi_{\mathrm{jam}}$ is approached), in agreement with results on other discrete models [14]. However, in the upper panel, the packing fraction at low intensities tends towards close-packing (the socalled dynamical transition referred to in [8]); this is at odds with the results of real experiment, which models with a greater degree of complexity [7] have been able to replicate. In the lower panel, which corresponds to our new model, we see tentative indications of an improvement in this respect vis-a-vis the old model; the packing fraction here asymptotes towards the jamming limit, rather than rising indefinitely towards the close-packing limit [7,30].

\section{Discussion}

We have presented in the above two models of shaken sandboxes; while their design was such that they would show identical behaviour in regimes where there were a finite density of voids, the modelling of the densely packed regime was completely 
distinct in each case. In the first case, the model reduced to a model of noninteracting spins, while in the second, the insistence that all allowed transitions minimised a suitably defined local packing fraction led in fact to an intricate coupling between the grains. This physically motivated interaction was extremely nonlocal as well as directional. In this way we were able to generate a model that, despite being one-dimensional, has an extremely complex ground-state structure, depending on the regularity of the grain shape. Our present investigations, to be published elsewhere [26], concern the effect of zero-temperature and finite-temperature tapping of this system; our preliminary studies indicate that for regularly shaped grains, strong metastability in the achievable ground states is observed. For irregularly shaped grains, as in reality, a far better packing is achievable, since orientations of irregularly shaped grains are much better able to fill space [33].

It is however a rather salutary exercise to see that despite the relative sophistication of the new model in its inclusion of non-trivial interactions, most of the qualitative behaviour of the packing fraction as a function of steady as well as annealed tapping, remains unchanged. We expect that quantitative features such as two-time correlation functions will be far more non-trivial in the second model than the first, although we expect their overall features to be rather similar. It is tempting to speculate that the directionality due to gravity (which leads to strongly non-Hamiltonian behaviour, since grain couplings are propagating down the pile) which unites both first and second models might well be the most important ingredient that is needed to describe such lattice-based models of shaken sand.

\section{References}

[1] Aradian A., Raphaël E., and de Gennes P.G. Thick surface flows of granular materials: Effect of the velocity profile on the avalanche amplitude. Phys. Rev. E, 60, 2009-2019 (1999).

[2] Barker G.C. and Mehta A. Vibrated powders - structure, correlations and dynamics. Phys. Rev. A, 45, 3435-3446 (1992).

[3] Barker G.C. and Mehta A. Transient phenomena, self-diffusion and orientational effects in vibrated powders. Phys. Rev. E, 47, 184-188 (1993).

[4] Barker G.C. and Mehta A. Avalanches at rough surfaces. Phys. Rev. E, 61, 6765-6772 (2000).

[5] Barker G.C. and Mehta A. Inhomogeneous relaxation in vibrated granular media: consolidation waves (2001). Phase Transitions, to appear; cond-mat/0010268.

[6] Berg J.M. and Mehta A. See contribution in this volume.

[7] Berg J.M. and Mehta A. Glassy dynamics of granular compaction: Sand on random graphs (2001). Phys. Rev. E, to appear; cond-mat/0108225.

[8] Berg J.M. and Mehta A. On random graphs and the statistical mechanics of granular matter (2001). Europhys. Lett., to appear; cond-mat/0012416.

[9] Berthier L., Cugliandolo L.F., and Iguain J.L. Glassy systems under time-dependent driving forces: Application to slow granular rheology. Phys. Rev. E, 63, 051302 (2001).

[10] Biham O., Middleton A.A., and Levine D. Self-organization and a dynamical transition in traffic-flow models. Phys. Rev. A, 46, R6124-6127 (1992).

[11] Boutreux T., Raphaël E., and de Gennes P.G. Surface flows of granular materials: A modified picture for thick avalanches. Phys. Rev. E, 58, 4692-4700 (1998). 
[12] Burridge R. and Knopoff L. Model and theoretical seismicity. Bull. Seis. Soc. Am., 57, 341-371 (1967).

[13] Carlson J.M. and Langer J.S. Mechanical model of an earthquake fault. Phys. Rev. A, 40, 6470-6484 (1989).

[14] Coniglio A. and Nicodemi M. The jamming transition of granular media. J. Phys. Cond. Matt., 12, 6601-6610 (2000).

[15] de Bruijn N.G. Sequences of zeros and ones generated by special production rules. Nederl. Akad. Wetensch. Proc. A, 84, 27-37 (1981).

[16] de Gennes P.G. Tapping of granular packs: A model based on local two-level systems. J. Coll. Int. Sci., 226, 1-4 (2000).

[17] Duneau M. and Katz A. Quasiperiodic patterns. Phys. Rev. Lett., 54, 2688-2691 (1985).

[18] Duneau M. and Katz A. Quasiperiodic patterns and icosahedral symmetry. J. Phys. (France), 47, 181-196 (1986).

[19] Edwards S.F. The role of entropy in the specification of a powder (1994). In Granular Matter: An Interdisciplinary Approach, ed. A. Mehta (Springer-Verlag, New York).

[20] Edwards S.F. and Grinev D.V. Statistical mechanics of vibration-induced compaction of powders. Phys. Rev. E, 58, 4758-4762 (1999).

[21] Elser V. Indexing problems in quasicrystal diffraction. Phys. Rev. B, 32, 4892-4898 (1985).

[22] Kalugin P.A., Kitayev A.Y., and Levitov L.S. $\mathrm{Al}_{0.86} \mathrm{Mn}_{0.14}$ : A six-dimensional crystal. J.E.T.P. Lett., 41, 145-149 (1985).

[23] Kalugin P.A., Kitayev A.Y., and Levitov L.S. Six-dimensional properties of $\mathrm{Al}_{0.86}$ $\mathrm{Mn}_{0.14}$ alloy. J. Phys. (France) Lett., 46, L601-L607 (1985).

[24] Kurchan J. Emergence of macroscopic temperatures in systems that are not thermodynamical microscopically: towards a thermodynamical description of slow granular rheology. J. Phys. Cond. Matt., 12, 6611-6617 (2000).

[25] Luck J.M., Godrèche C., Janner A., and Janssen T. The nature of the atomic surfaces of quasiperiodic self-similar structures. J. Phys. A, 26, 1951-1999 (1993).

[26] Luck J.M., Stadler P.F., and Mehta A. (2001).

[27] Mehta A. and Barker G.C. Vibrated powders - a microscopic approach. Phys. Rev. Lett., 67, 394-397 (1991).

[28] Mehta A. and Barker G.C. Disorder, memory and avalanches in sandpiles. Europhys. Lett., 27, 501-506 (1994).

[29] Mehta A. and Barker G.C. Glassy dynamics of granular compaction. J. Phys. Cond. Matt., 12, 6619-6628 (2000).

[30] Nowak E.R., Knight J.B., Ben-Naim E., Jaeger H.M., and Nagel S.R. Density fluctuations in vibrated granular materials. Phys. Rev. E, 57, 1971-1982 (1998).

[31] Nowak E.R., Knight J.B., Povinelli M., Jaeger H.M., and Nagel S.R. Reversibility and irreversibility in the packing of vibrated granular material. Powder Technology, 94, 79-83 (1997).

[32] Stadler P.F., Mehta A., and Luck J.M. Shaking a box of sand (2001). Condmat/0103076.

[33] Torquato S. and Chaikin P. (unpublished).

[34] Villarruel F.X., Lauderdale B.E., Mueth D.M., and Jaeger H.M. Compaction of rods: Relaxation and ordering in vibrated, anisotropic granular material. Phys. Rev. E, 61, 6914 (2000).

[35] Weeks E.R., Crocker J.C., Levitt A.C., Schofield A., and Weitz D.A. Three-dimensional direct imaging of structural relaxation near the colloidal glass transition. Science, 287, 627-631 (2000). 